\title{
The reaction of 2-chloro-1-methoxynaphthalene and 2- bromobiphenylene with 3-cyanophthalides under aryne-forming conditions: convenient synthesis of annular polycyclic aromatic compounds
}

\author{
Yingchun Lu, Cheng-Han Wu, and Ed Biehl* \\ Chemistry Department, Southern Methodist University, Dallas, TX 75275 \\ E-mail: ebiehl@mail.smu.edu
}

(received 18 Jul 02; accepted 05 May 03; published on the web 09 May 03)

\begin{abstract}
2-Bromobiphenylene and 2-chloronaphthalene react with 3-cyanophthalides in the presence of LDA or LiTMP to give functionalized benzo[3,4]cyclobuta[1,2-b]anthracene-6,11-dione and naphthacene-5,12-dione, respectively.
\end{abstract}

Keywords: Benzyne, aryne, cycloaddition, nucleophilic addition, polycyclic aromatic compounds

\section{Introduction}

Biphenylenes have recently been studied extensively because they can serve as units of new carbon allotropes and can function as spacers and building blocks for functionalized organic materials. ${ }^{1}$ One of the more important syntheses of functionalized biphenyls involves the intramolecular coupling of benzo-annulated zinca-cyclopentadiene intermediates prepared from 2,2'-diaryls with $\mathrm{ZnCl}_{2}{ }^{2}$ During the course of our studies on the synthetic use of aryne reaction, we have prepared a wide variety of functionalized polycyclic quinones from the reaction of haloarenes with 3-lithiophthalides in the presence of sterically hindered bases such as LDA and LiTMP. $^{3}$ It occurred to us that 2-bromobiphenylene might undergo similar reactions to give functionalized annulated biphenyls. Although 2-bromobiphenylene can, in principle give two benzynes, i.e., 1,2-dehydro- and 2,3-dehydro-biphenylene, our calculations have shown the 2,3-intermediate to be 4.47 $\mathrm{kcal} / \mathrm{mol}$ more stable than the 1,2- isomer. The relative stability of 1,2- and 2,3-dehydrobiphenyl were calculated with complete optimization of all geometric variables using the standard AM1 procedure ${ }^{4}$ incorporated in version 3.1 of the Spartan Package. ${ }^{5}$ Furthermore, the latter, being symmetric, would give a single aryne product. 
Along similar lines, we decided to investigate also the reaction of 2-chloro-1-methoxynaphthalene with certain 3-cyano-1 $(3 H)$ isobenzofuranones (3-cyanophthalides). The well-proven meta-directing effect of the methoxy group ${ }^{6}$ should also afford a single aryne product.

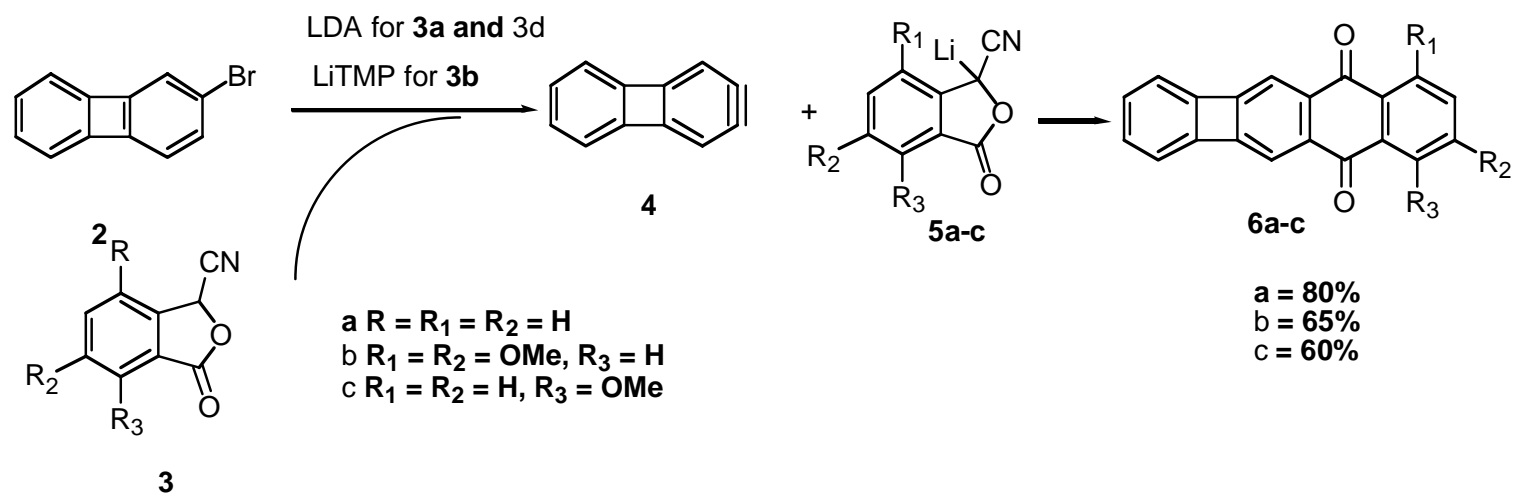

\section{Scheme 1}

\section{Results and Discussion}

Our expectations were indeed realized, and are illustrated in Scheme 1 and Scheme 2. As shown in Scheme 1, 2-bromobiphenylene (2) reacted and the 3-cyanophthalides (3a-c) reacted with LDA or LiTMP, respectively, to produce 2,3-dehydrobiphenylene (4) and lithiated 3-cyanophthalides (5a-c). These intermediates then underwent cycloaddition to give the corresponding benzo[3,4]cyclobuta[1,2-b]anthracene-6,11-dione (6a-c) in $60-80 \%$ yields after the usual workup. The benzyne precursor, 2-bromobiphenylene (2) was prepared by the bromination of biphenylene (1) with DBU in the presence of $\mathrm{HgCl}_{2}$ in $75 \%$ yield. ${ }^{7}$ This method is superior to simple bromination which gives 2 in only $50 \%$ along with some polybromides. ${ }^{8}$ The 3 -cyanophthalides (3a-c) were on hand from previous studies and IR spectra were consistent with the proposed structures.

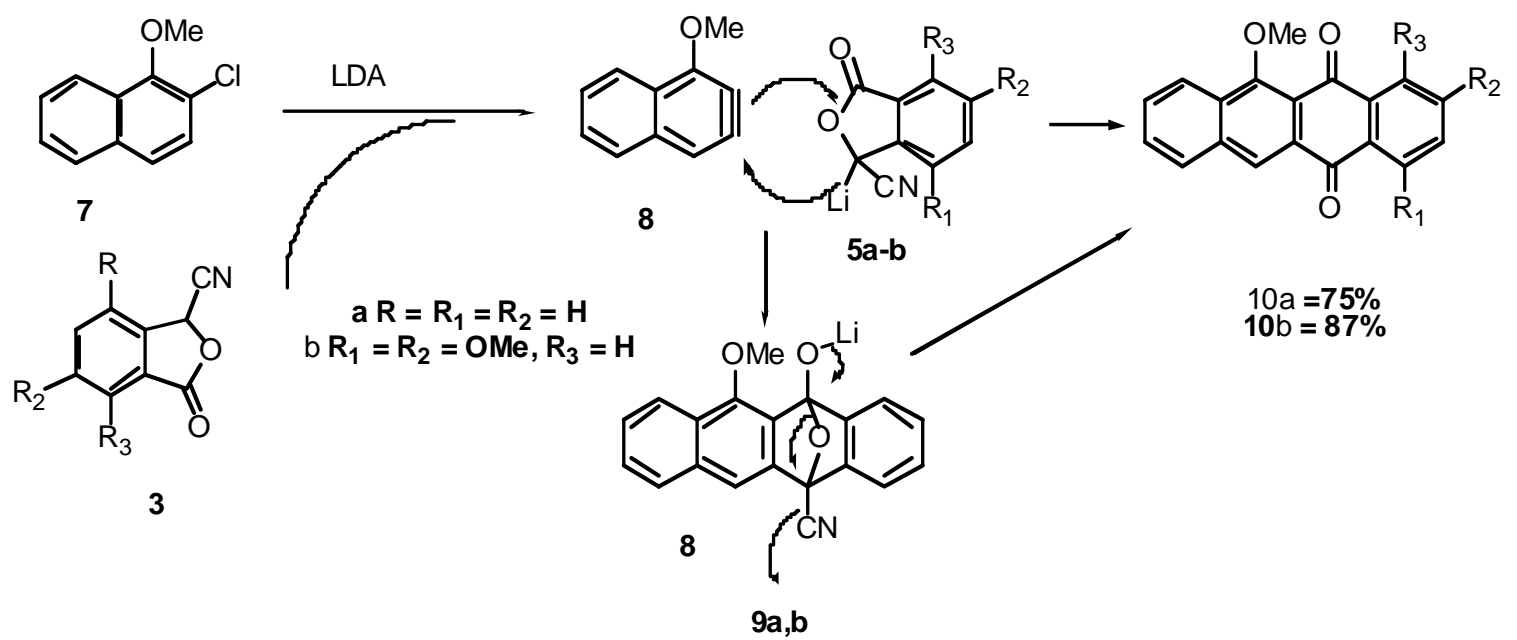

\section{Scheme 2}


As shown in Scheme 2, 1-methoxynaphthacene-5,12-dione (10a) and 1,3,11-trimethoxy-naphthacene5,12-dione (10b), respectively, were obtained in 75 and 87\% yields from the reaction of 2-chloronaphthalene (7) and the 3-cyanophthalides (3a and 3b). The regioselective addition to 1-methoxy-2,3-dehydro-naphthalene (8) was clearly shown in the case of $\mathbf{5 b}$ to give a single adduct (9b). Obviously, no regiochemistry is involved in the case of the unsubstituted lithiated nitrile 5a. The IR, $1 \mathrm{H} \mathrm{NMR},{ }^{13} \mathrm{C}-\mathrm{NMR}$, and mass spectra were consistent with the proposed structures.

We next treated 7 with $\alpha$-cyano-o-tolunitrile 11 and LDA, expecting aryne 8 and $\alpha$-lithio- $\alpha$-cyano-otolunitrile (12) to undergo $[2+4\}$ cycloaddition ${ }^{9}$ to give the aminonaphthacene 13. However, as shown in Scheme 3, this reaction proceeded by a tandem addition-rearrangement pathway ${ }^{10}$ in which $\mathbf{8}$ and $\mathbf{1 2}$ reacted via a [2+2]-cycloaddition pathway to the benzocyclobutenium adduct (14). Intermediate 14 then opened up to give the rearranged product, 3-(2'-cyanobenzyl)-1-methoxy-2-naphthalene-carbonitrile (15) in 40\% yield, after quenching.

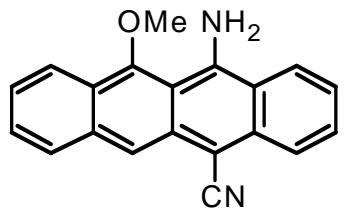

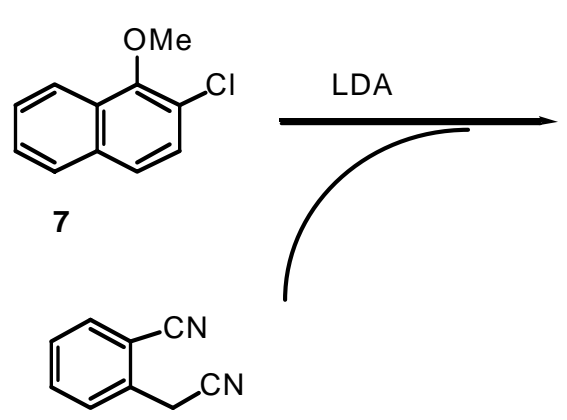

11
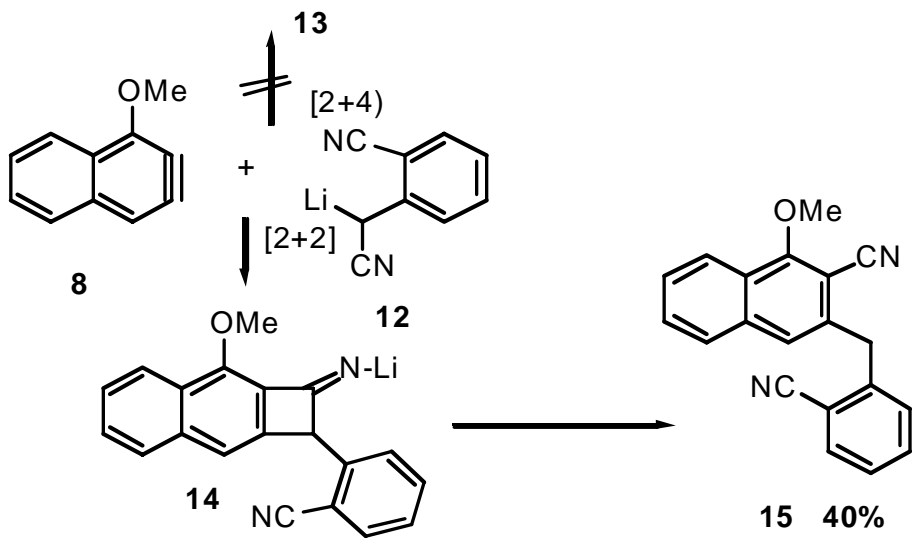

\section{Scheme 3}

In conclusion, we have shown that 2,3-dehydrobiphenylene and 1-methoxy-2,3-dehydronaphthalene can serve as valuable intermediates in the synthesis of functionalized benzo[3,4]cyclobuta[1,2-b]anthracene-6,11dione and naphthacene-5,12-dione, respectively.

\section{Experimental Section}

General Procedures. Melting points were taken on a Mel-Temp apparatus and are not corrected. NMR spectra were recorded on a $400 \mathrm{MHz}$ spectrometer: chemical shifts were related to TMS as internal standard. 
Chemicals were purchased from commercial sources. LDA and BuLi were purchased as solutions in hexanes. The glassware heated at $125^{\circ} \mathrm{C}$ in an oven overnight prior to use. Benzyne reactions were done under an atmosphere of dry $\mathrm{O}_{2}$-free $\mathrm{N}_{2}$ contained in a balloon possessing a needle protruding through a rubber septum attached to one of the reaction flask necks.

Biphenylene (1), ${ }^{2}$ 2-bromobiphenylene (2), ${ }^{7}$ and 2-chloro-1-methoxynaphthalene (7) ${ }^{11}$ were prepared by literature procedures. The 3-cyanophthalides $(\mathbf{4 a}-\mathbf{c})$ were available from previous studies.

General procedure for the reaction of haloarenes (2 and 7) with 3-cyanophthalides (4a-c). In a flamedried flask flushed with $\mathrm{N}_{2}$, LDA (15 mmol) was prepared by adding $6 \mathrm{~mL}$ of $n$-BuLi (2.5 M in hexanes) to a solution containing diisopropylamine $(15 \mathrm{mg}, 15 \mathrm{mmol})$ in $\mathrm{THF}(30 \mathrm{~mL})$ at $-70^{\circ} \mathrm{C}$. After stirring for $10 \mathrm{~min}, 5$ mmol of the appropriate nitrile (4a-c) in $30 \mathrm{~mL}$ of THF was added and the temperature allowed to warm to -40 ${ }^{\circ} \mathrm{C}$. At this point, $5 \mathrm{mmol}$ of the haloarene ( 2 or 7 ) in $30 \mathrm{~mL}$ of THF was added over a period of 20 min while maintaining the temperature between -30 to $-40{ }^{\circ} \mathrm{C}$. After the addition of the haloarene, the resulting mixture was allowed to warm to r.t. where it was stirred for an additional $3 \mathrm{~h}$. The resulting dark reddish solution was quenched with $30 \mathrm{~mL}$ of sat. $\mathrm{NH}_{4} \mathrm{Cl}$ and the THF removed by rotary evaporator. The residue was extracted with $\mathrm{CH}_{2} \mathrm{Cl}_{2}(3 \times 20 \mathrm{~mL})$. The $\mathrm{CH}_{2} \mathrm{Cl}_{2}$ fractions were combined, washed with $25 \mathrm{ml}$ of $5 \% \mathrm{HCl}$, then brine, then dried $\left(\mathrm{Na}_{2} \mathrm{SO}_{4}\right)$ and concentrated (rotary evaporator) to provide a dark viscous liquid. The liquid was purified by flash chromatography (silica gel) using hexane/acetone (19:1) as eluent to give a solid product which was further purified by recrystallization from $\mathrm{CH}_{2} \mathrm{Cl}_{2}$-hexane. The physical properties of the products are given below.

Benzo[3,4]cyclobuta[1,2-b]anthracene-6,11-dione (8a). Yellow powder, mp 218-219 ${ }^{0} \mathrm{C}$. IR (KBr) $v 1684$ $\mathrm{cm}^{-1} .{ }^{1} \mathrm{H}$ NMR $\left(\mathrm{CDCl}_{3}\right) \delta 6.81(\mathrm{~d}, \mathrm{~J}=\mathrm{Hz}, 2 \mathrm{H}), 6.92(\mathrm{~m}, 2 \mathrm{H}), 7.32(\mathrm{~d}, \mathrm{~J}=8.0 \mathrm{~Hz}, 1 \mathrm{H}), 7.73(\mathrm{~m}, 2 \mathrm{H}), 7.79$ $(\mathrm{d}, \mathrm{J}=8.1 \mathrm{~Hz}, 1 \mathrm{H}), 8.23(\mathrm{~m}, 1 \mathrm{H}), 8.32(\mathrm{~m}, 1 \mathrm{H}) .{ }^{13} \mathrm{C} \mathrm{NMR}\left(\mathrm{CDCl}_{3}\right) \delta 119.2,120.1,121.9,123.6,126.8$, 127.7, 130.8, 130.9, 131.0, 131.8, 133.7, 133.8, 134.1, 149.3, 151.1, 153.5, 158.2, 181.8, 182.2. Anal. Calcd for $\mathrm{C}_{20} \mathrm{H}_{10} \mathrm{O}_{2}$ : C, 85.09; $\mathrm{H}, 3.57$. Found: 85.15; H, 3.66\%.

1-Methoxybenzo[3,4]cyclobuta[1,2-b]anthracene-6,11-dione (8b). Yellow powder, mp 238-240 ${ }^{0} \mathrm{C}$. IR $(\mathrm{KBr}) \vee 1667 \mathrm{~cm}^{-1} .{ }^{1} \mathrm{H}$ NMR $\left(\mathrm{CDCl}_{3}\right) \delta 4.01(\mathrm{~s}, 3 \mathrm{H}), 6.91(\mathrm{~m}, 4 \mathrm{H}), 7.31(\mathrm{~d}, \mathrm{~J}=8.1,1 \mathrm{H}), 7.41(\mathrm{~s}, 1 \mathrm{H}), 7.52$ $(\mathrm{s}, 1 \mathrm{H}), 7.7(\mathrm{t}, \mathrm{J}=8.1 \mathrm{~Hz}, 1 \mathrm{H}), 7.94(\mathrm{~d}, \mathrm{~J}=8.1 \mathrm{~Hz}, 1 \mathrm{H}), 8.2(\mathrm{~m}, 1 \mathrm{H}), 8.3(\mathrm{~m}, 1 \mathrm{H}) .{ }^{13} \mathrm{C} \mathrm{NMR}\left(\mathrm{CDCl}_{3}\right) \delta$ 56.6, 113.0, 113.9, 117.8, 119.6, 119.7, 119.8, 1303, 130.4, 134.8, 135.2, 137.0, 137.9, 149.6, 156.2, 157.3, 160.1, 171.2, 182.1, 182.9. Anal. Calcd for $\mathrm{C}_{21} \mathrm{H}_{12} \mathrm{O}_{3}$ : C, 80.76; H, 3.87. Found: 80.87; H, 3.90\%.

1,3-Dimethoxybenzo[3,4]cyclobuta[1,2-b]anthracene-6,11-dione (8c). Yellow powder, $\mathrm{mp} 208-210{ }^{0} \mathrm{C}$. IR $(\mathrm{KBr}) \vee 1682 \mathrm{~cm}^{-1} .{ }^{1} \mathrm{H}$ NMR $\left(\mathrm{CDCl}_{3}\right) \delta 4.0(\mathrm{~s}, 6 \mathrm{H}), 6.8(\mathrm{~d}, J=8.0 \mathrm{~Hz}, 2 \mathrm{H}), 6.9(\mathrm{~m}, 2 \mathrm{H}), 7.3(\mathrm{~d}, J=8 \mathrm{~Hz}, 1$ $\mathrm{H}), 7.7(\mathrm{~m}, 2 \mathrm{H}), 7.8(\mathrm{~d}, \mathrm{~J}=8 \mathrm{~Hz}, 1 \mathrm{H}), 8.2(\mathrm{~m}, 1 \mathrm{H}), 8.3(\mathrm{~m}, 1 \mathrm{H}) .{ }^{13} \mathrm{C} \mathrm{NMR}\left(\mathrm{CDCl}_{3}\right) \delta 130.9,131.0,131.8$, 133.7, 133.8, 134.1, 149.3, 151.1, 153.5, 158.2, 181.8, 182.2. Anal. Calcd for $\mathrm{C}_{20} \mathrm{H}_{10} \mathrm{O}_{2}$ : C, 85.09; H, 3.57. Found: $85.15 ; \mathrm{H}, 3.66 \%$.

1-Methoxynaphthacene (10a). colorless prisms, mp 283-284 ${ }^{\circ} \mathrm{C}$. IR $(\mathrm{KBr}) y 1677 \mathrm{~cm}^{-1} .{ }^{1} \mathrm{H}$ NMR $\delta 4.21(\mathrm{~s}, 3$ H), $7.82(\mathrm{~m}, 4 \mathrm{H}), 8.10(\mathrm{~m}, 1 \mathrm{H}), 8.33(\mathrm{~m}, 2 \mathrm{H}), 8.44(\mathrm{~m}, 1 \mathrm{H}), 8.70(\mathrm{~s}, 1 \mathrm{H}) .{ }^{13} \mathrm{C}$ NMR $\delta 63.0,120.0,124.7$, 125.8, 127.0, 127.5, 129.5, 130.0, 130.3, 130.9, 132.0, 133.5, 133.6, 134.3, 135.9, 126.0, 160.0, 182.2, 183.2. Anal. Calcd for $\mathrm{C}_{19} \mathrm{H}_{12} \mathrm{O}$ : C, 79.16; H, 4.20. Found: C, 79.31; H, 4.26\%. 
1,3,11-Trimethoxynaphthacene (10b). light yellow powder, mp $216-217^{\circ} \mathrm{C}$. IR (KBr) $1667 \mathrm{~cm}^{-1} .{ }^{1} \mathrm{H}$ NMR $\left(\mathrm{CDCl}_{3}\right) \delta 3.91(\mathrm{~s}, 3 \mathrm{H}), 4.01(\mathrm{~s}, 3 \mathrm{H}), 4.12(\mathrm{~s}, 3 \mathrm{H}), 6.72(\mathrm{~d}, \mathrm{~J}=4.2 \mathrm{~Hz}, 1 \mathrm{H}), 7.50(\mathrm{~d}, \mathrm{~J}=4.1 \mathrm{~Hz}, 1 \mathrm{H}), 7.72$ $(\mathrm{m}, 2 \mathrm{H}), 8.13(\mathrm{~m}, 1 \mathrm{H}), 8.41(\mathrm{~m}, 1 \mathrm{H}), 8.58(\mathrm{~m}, 1 \mathrm{H}) .{ }^{13} \mathrm{C} \mathrm{NMR}\left(\mathrm{CDCl}_{3}\right) \delta 56.0,56.6,63.1,103.1,104.4$, 116.7, 120.0, 124.5, 125.2, 128.9, 130.1, 131.1, 132.5, 136.3, 140.0, 159.2, 162.3, 164.9, 181.2, 182.4. Anal. Calcd for $\mathrm{C}_{21} \mathrm{H}_{16} \mathrm{O}_{3}$ : C, 72.41; H, 4.63. Found: $\mathrm{C}, 72.45 ; \mathrm{H}, 4.70 \%$.

3-(2'-Cyanobenzyl)-1-methoxy-2-naphthalenecarbonitrile (15). Colorless crystals, mp $142-143{ }^{\circ} \mathrm{C}$. IR $(\mathrm{KBr}) \vee 2223 \mathrm{~cm}^{-1} .{ }^{1} \mathrm{H}$ NMR $\left(\mathrm{CDCl}_{3}\right) \delta 4.21(\mathrm{~s}, 3 \mathrm{H}), 4.51(\mathrm{~s}, 2 \mathrm{H}), 7.30-7.80(\mathrm{~m}, 8 \mathrm{H}), 8.21(8.21(\mathrm{~d}, \mathrm{~J}=8.1$ $\mathrm{Hz}, 1 \mathrm{H}) .{ }^{13} \mathrm{C} \mathrm{NMR}\left(\mathrm{CDCl}_{3}\right) \delta 38.5,63.0,101.3,113.1,122.8,124.2,126.1,127.0,127.4,127.8,130.0,130.2$, 133.1, 133.2, 136.0, 136.4, 142.5, 162.5. Anal. Calcd for $\mathrm{C}_{20} \mathrm{H}_{14} \mathrm{~N}_{2} \mathrm{O}: \mathrm{C}, 80.52, \mathrm{H}, 4.73, \mathrm{~N}, 9.39$. Found: $\mathrm{C}$, $80.55 ; \mathrm{H}, 4.74 ; \mathrm{N}, 9.44 \%$.

\section{Acknowledgements}

Generous financial assistance from the Robert Welch Foundation, Houston, Texas is greatly appreciated.

\section{References}

1. Iyoda, M.; Kabir, S. M. H.; Vorasingha, A.; Kuwatani, Y.; Yosida, M. Tetrahedron Lett. 1998, 39, 5393, and references therein.

2. Kabir, S. M. H.; Haseqawa, M.; Kuwatani, Y.; Yoshida, M.; Matsuyama, H.; Iyoda, M. J. Chem. Soc. Trans 1 2001, 159.

3. Khanapure, S. P.; Biehl, E. R. Acc. Chem. Res. 1989, 22, 275.

4. Dewar, M. J. S.; Zoebisch, E. G.; Healy, E. F.; Stewart, J. J. P. J. Am. Chem. Soc. 1985, 107, 3902.

5. SPARTAN version 3.1. Wavefunction, Inc. 18401 Von Karman, \#370, Irvine, CA 92715 USA. C1993.

6. $\quad$ Roberts, J. D.; Vaughan, C. W.; Carlsmith, L. A.; Semenov, D. J. Org. Chem. 1998, 63, 2451.

7. Muathen, H. A. J. Org. Chem. 1992, 57, 2740.

8. Cava, M. P.; Mitchell, M. J., Cyclobutadiene and Related Compounds; Academic Press: New York, 1967.

9. Han, W.; Lu, Y.; Zhao, H.; Dutt, M.; Biehl, E. R. Synthesis 1996, 59.

10. Pansegrau, P. D.; Rieker, W. F.; Meyers, A. I. J. Am. Chem. Soc. 1988, 110, 7148.

11. Smithwick, Jr., E. L.; Shuman, R. T. Synthesis 1974, 8, 582. 